\title{
Biomechanics of pelvic organ prolapse
}

\author{
REN Shuang $^{1}$, XIE Bing $^{2}$, WANG JianLiu ${ }^{2} \&$ RONG QiGuo ${ }^{1 *}$ \\ ${ }^{1}$ College of Engineering, Peking University, Beijing 100871, China; \\ ${ }^{2}$ Department of Obstetrics \& Gynecology, Peking University People's Hospital, Peking University, Beijing 100871, China
}

Received May 26, 2014; accepted October 14, 2014; published online January 4, 2015

Citation: Ren S, Xie B, Wang JL, Rong QG. Biomechanics of pelvic organ prolapse. Sci China Life Sci, 2015, 58: 218-220, doi: 10.1007/s1 1427-014-4767-2

Dear Editor,

Pelvic organ prolapse (POP), caused by damage of the support system in the pelvic floor, is downward descent of female pelvic organs, resulting in protrusion of the vagina, uterus, or both. It has been reported recently that POP has a prevalence of $3 \%-6 \%$ when defined by symptoms and up to $50 \%$ based on a vaginal examination [1]. POP is basically divided into anterior vaginal wall prolapse and posterior vaginal wall prolapse. The most common type of POP is anterior vaginal wall prolapse, clinically known as the cystocele [2]. Most POP patients have damages in the support muscles or ligaments or both of them. Biomechanical nature of POP is of great significance for clinical prevention and treatments.

Risk factors for POP include vaginal delivery, chronic increased intraabdominal pressure caused by obesity, chronic respiratory disease and/or related cough, chronic constipation, repetitive occupational activities, and pregnancy [3,4]. However, the mechanism of POP remains unclear.

Delancey [5] divided the support system of the pelvic floor into three levels (Figure 1). The first level is provided by the cardinal and uterosacral ligaments. The second level includes the "hammock-like" levator ani muscle and pelvic fascia that covers the muscles and provides reinforcements. The third level is the distal support by the perineal body, counteracting the downward gravity of the organs.

*Corresponding author (email: qrong@pku.edu.cn)

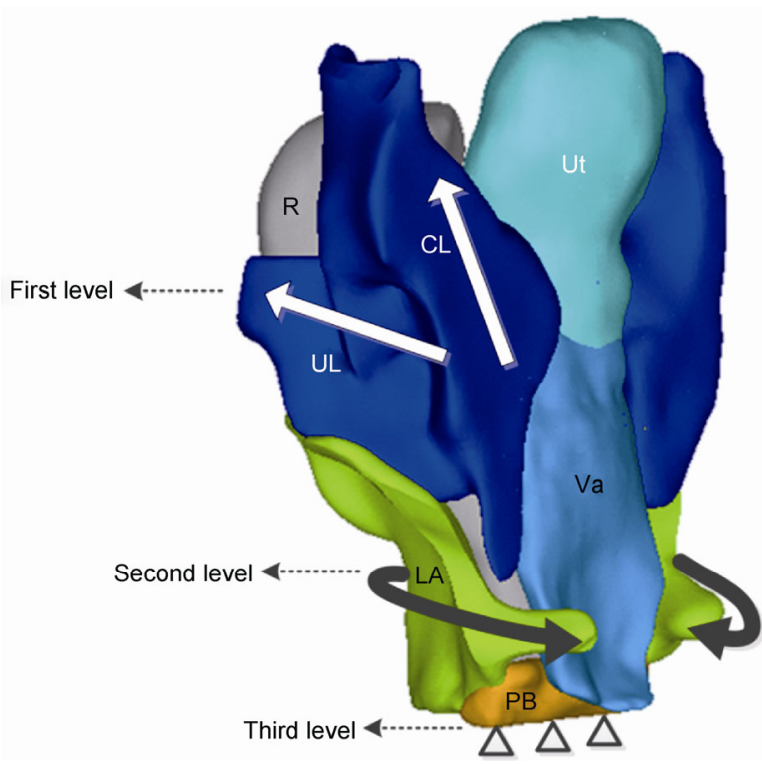

Figure 1 (color online) Three levels of the support system of the normal pelvic floor. R, rectum; CL, cardinal ligament; UL, uterosacral ligament; LA, levator ani muscle; Ut, uterus; Va, vagina; PB, perineal body. The model was reconstructed based on magnetic resonance images from a female volunteer (55 years old, para 2; body mass index (BMI) 20.96 $\mathrm{kg} \mathrm{m}^{-2}$ ).

When the levator ani muscle contracts, it will close the urogenital hiatus through which prolapse may occur, making a closed pelvic so that the viscera are under no differential pressure. In this case, the levator ani muscle stands most of the support and the ligaments sustain smaller loads [5]. When the levator ani muscle damages or relaxes, the liga- 
ments start to carry the uterus. However, if the viscoelastic ligaments keep being stretched for a long time, they will finally get damaged, and prolapse will occur [6].

In clinical studies, medical researchers usually speculate the possible causes of POP from observed symptoms, namely from function to structure, a bottom-up way. On the contrary, it can also be studied in top-down direction, namely studying the effect of the possible damage of the pelvic supporting structures. Finite element (FE) method is a non-invasive and repetitive numerical simulation method which makes it effective for this kind of studies. Chen et al. [7] established a two-dimensional (2D) biomechanical model of the anterior vaginal wall and its support system. The results showed that if the pubovisceral impairment reached at a certain level, the anterior vaginal wall would prolapse; at $60 \%$ pubovisceral muscle impairment, a $90 \%$ impairment of apical support caused the prolapse to increase from 0.3 to $1.9 \mathrm{~cm}$. In 2009, a three-dimensional (3D) finite element model of the anterior vaginal wall and its support system were developed by Chen et al. [8]. Effects of the apical impairment and/or levator ani muscle impairment on prolapse size were evaluated. It showed that cystocele size was more sensitive to levator ani muscle impairment than apical impairment.

The application of FE analysis in modeling and simulation of the pelvic support system, however, is still in its beginning stage. Some basic problems need to be clarified in simulating the occurrence, development and prognosis of POP.

The first problem is to establish a numerical model that includes all pelvic supporting structures and has high geometrical similarities. The anatomical structures of POP are too complex for FE modeling and simulation. Therefore, some structural simplifications had been done in previous FE studies. The anterior vaginal wall was usually modeled as a separated part from the posterior compartment, which was entirely made up of the posterior vaginal wall and rectum. In addition, the ligaments were simulated by passive elastic springs [7] or 3D truss elements [8]. The oversimplification made it impossible to evaluate the shear stress in the lateral vaginal walls, which is, however, eagerly needed clinically. Besides, the previous FE studies did not consider the closure or opening of vagina, namely the contacting of the anterior and posterior vaginal walls, a crucial element in preventing prolapse by friction [9]. Future FE models of the pelvic floor should include a vaginal lumen, instead of a solid vagina or a separated anterior vaginal wall. Up to now, there is no FE study for pelvic organ prolapse which uses realistic anatomies for all the support structures.

The second problem is how to set the realistic loading and boundary conditions. The boundary conditions should be defined based on the functional anatomy of the pelvic floor. In previous studies, the pelvis was usually assumed to be fixed. The origins of the levator ani muscle and the cardinal and uterosacral ligaments from the pelvic wall were set stationary in 2D simulation [7], rotatable but not translatable in 3D simulation [8]. The problem located in the perineal body, which was usually assumed to be stationary [7]. In Chen's 3D simulation of POP, the distal vagina was set fixed since there is no support of the perineal body in this model. However, the distal vagina was sometimes observed moving downward clinically [8]. According to the anatomy, the lower portion of the perineal body is attached to the ischiopubic rami by superficial transverse perineal muscles. Therefore, future FE studies should consider the support of the perineal body.

The existing FE studies of POP [7,8] applied a constant pressure on the anterior vaginal wall to simulate Valsalva maneuver. However, abdominal pressure is filling the whole pelvic floor in reality. When the support structures are too weak to hold the organs in situ, the vaginal wall will protrude through the introitus, exposing the unsupported prolapsed region of the vagina due to the differential pressure between the abdominal and atmospheric pressures. This will cause tension in the vaginal wall, leading to descent of the apical vagina. The higher the differential pressure is, the more descending the vagina becomes. This is the feed-back mechanism that Chen proposed [8]. Therefore, the abdominal and atmosphere pressures will affect loads on the ligaments and muscles. In further studies, the abdominal and atmosphere pressures should be considered more realistically.

The third problem is how to choose and validate the material models: (i) material properties of pelvic muscles and ligaments were usually not validated in previous studies; (ii) simulating the active behaviors of the levator ani muscle is still a challenge; (iii) complex material models, such as viscoelasticity and hyperelasticity, will greatly increase the computational difficulties, such as long solving time and difficulties in convergence. Impairments of muscles and ligaments can be modeled by decreasing stiffness [8]. Detachments of the muscle or fascia can be simulated by setting the elastic modulus of the attached elements to a small value. More studies about human pelvic muscles and ligaments are required to improve the accuracy of FE analysis.

FE analysis is a powerful tool in studying POP, but still needs essential improvements in further studies.

This work was supported in part by the Seeding Grant for Medicine and Engineering Sciences of Peking University (2014-ME-01/02...22).

1 Barber MD, Maher C. Epidemiology and outcome assessment of pelvic organ prolapse. Int Urogynecol J, 2013, 24: 1783-1790

2 Hendrix S. Pelvic organ prolapse in the women's health initiative: gravity and gravidity. Am J Obstet Gynecol, 2002, 186: 1160-1166

3 O'Dell KK, Morse AN. It's not all about birth: biomechanics applied to pelvic organ prolapse prevention. J Midwifery Womens Health, 2008, 53: 28-36

4 Bump RC, Norton PA. Epidemiology and natural history of pelvic floor dysfunction. Obstet Gyn Clin N Am, 1998, 25: 723-746 
5 Delancey JOL. The anatomy of the pelvic floor. Curr Opin Obstet Gyn, 1994, 6: 313-316

6 Ashton-Miller JA, Delancey JOL. Functional anatomy of the female pelvic floor. Ann Ny Acad Sci, 2007, 1101: 266-296

7 Chen L, Ashton-Miller JA, Hsu Y, DeLancey JO. Interaction among apical support, levator ani impairment, and anterior vaginal wall prolapse. Obstet Gynecol, 2006, 108: 324-332
8 Chen L, Ashton-Miller JA, DeLancey JO. A 3D finite element model of anterior vaginal wall support to evaluate mechanisms underlying cystocele formation. J Biomech, 2009, 42: 1371-1377

9 Larson KA, Luo J, Guire KE, Chen L, Ashton-Miller JA, DeLancey JO. 3D analysis of cystoceles using magnetic resonance imaging assessing midline, paravaginal, and apical defects. Int Urogynecol J, 2012, 23: 285-293

Open Access This article is distributed under the terms of the Creative Commons Attribution License which permits any use, distribution, and reproduction in any medium, provided the original author(s) and source are credited. 